\title{
Measurement Error Effect on the Power of Control Chart for Doubly Truncated Normal Distribution under Standardization Procedure
}

\author{
Ashit B. Chakraborty \\ St. Anthony's College, Shillong, Meghalaya, India \\ abc_sac@rediffmail.com \\ Anwer Khurshid \\ Department of Mathematical and Physical Sciences, College of Arts and Science \\ University of Nizwa, P. O. Box 33, PC 616, Birkat Al Mouz, Oman \\ anwer@unizwa.edu.om; anwer_khurshid@yahoo.com
}

\begin{abstract}
Researchers in various quality control procedures consider the possibility of measurement error effect on the power of control charts as an important issue. In this paper the effect of measurement errors on the power curve of standardization procedure will be studied for doubly truncated normal distribution. A method of obtaining the expression of the power of control chart for doubly truncated normal distribution is being proposed. The effect of truncation will be shown accordingly. To study the sensitivity of the monitoring procedure, average run length ( $A R L$ ) is also considered.
\end{abstract}

Keywords: Power, Doubly-truncated normal distribution (DTND), Measurement error, Average run length ( $A R L)$.

\section{Introduction}

The truncated distributions are quite important and widely used by researchers. Kendall and Stuart (1979) have discussed an interesting example of a practical application of various types of truncated distributions: Suppose the underlying variate $x$ simply cannot be observed in part or parts of its range. For example, if $x$ is the distance from the centre of a vertical circular target for fixed radius $R$ on a shooting target, we can only observe $x$ for shots actually hitting the target. If we have no knowledge of how many shots were fired at the target (say $n$ ) we simply have to accept $m$ values of $x$ observed on the target as coming from a distribution ranging from 0 to $R$. We then say that $x$ is truncated on the right at $R$. Similarly, if we define $Y$ in this example as the distance of a shot from the vertical line through the centre of the target, $Y$ may range from $-R$ to $R$ and its distribution is doubly truncated. Similarly, we may have a variate truncated on the left i.e. if observations below a certain value are not recorded.

The normal distribution in statistical quality control (process control) arises because observations of a measurable quality characteristic vary under large number of small random disturbances. And it varies similarly on both sides of the average. $\bar{X}$ and $R$ charts are the typical statistical control charts for variables where normal distribution is employed to control the location and dispersion of the characteristic under consideration. However, there are some practical situations where truncated form of a normal distribution may provide better approximation to the actual distribution. 
Since Cohen's initial contribution in the 1940s, several studies have looked at the various aspects of the truncated normal distributions. See, for example, Cohen (1949, 1950, 1991), Schneider (1986), Johnson (2001), Johnson and Thomopoulos, (2004), Horrace (2015). A number of authors used truncated normal distribution in statistical quality control. Rai (1966) constructed CUSUM for truncated normal distribution. Schneider (1985) studied the performance of the standard sampling plans when the variable understudy is distributed as truncated normal distribution. He showed how variable sampling plans can be designed when the truncation points and the normal population standard deviation $\sigma$ are known. The case of unknown $\sigma$ was also discussed by him. Kakoty and Chakraborty (1990) studied continuous inspection sampling plan based CUSUM chart for controlling the mean of a doubly truncated normal distribution. They used Fredholm integral equation to obtain Type C-OC curve. Recently, Cox (2009) applied truncated normal distribution to derive integral equations for the average run length (ARL), a key measure of the performance of a control chart.

In this paper the effect of errors on the power curve of standardization procedure will be studied for DTND and the effect of truncation will be shown accordingly along with the calculation of average run length $(A R L)$.

\section{Measurement Error}

Measurement errors, which often exist in practice, may considerably affect the performance of control charts (Ryan, 2011). The sources of error may be due to inherent variability in the process and the error due to measurement instrument. The efficiency and the ability of the control chart to detect the shift of the process level will be affected if the measurement error is large relative to the process variability (Chakraborty and Khurshid 2013 b). Sankle et al. (2012) discussed the cumulative sum control charts for the truncated normal distribution under measurement error. Chakraborty and Khurshid (2013 a) and Khurshid and Chakraborty (2014) also studied measurement error effect on the power of control charts for various truncated distributions. For the consequences of measurement error on the actual performance of various control charts see Chakraborty and Khurshid (2013 b) and references therein.

\section{Doubly Truncated Normal Distribution}

A random variable $X$ has doubly truncated normal distribution if its probability density function is given by

$$
\begin{aligned}
f(x ; \mu, \sigma) & =\frac{1}{\sigma \sqrt{2 \pi}} \exp \left\{-\frac{1}{2}\left(\frac{x-\mu}{\sigma}\right)^{2}\right\}\left[\frac{1}{\sigma \sqrt{2 \pi}} \int_{A}^{B} \exp \left\{-\frac{1}{2}\left(\frac{x-\mu}{\sigma}\right)^{2}\right\} d x\right]^{-1} ; A \leq X \leq B \\
& =\frac{1}{\sigma} \phi\left(\frac{x-\mu}{\sigma}\right)\left[\Phi\left(\frac{B-\mu}{\sigma}\right)-\Phi\left(\frac{A-\mu}{\sigma}\right)\right]^{-1}
\end{aligned}
$$

where $\phi(\cdot)$ and $\Phi(\cdot)$ are probability density function and cumulative distribution function of standardized normal variate respectively. If $A$ is replaced by $-\infty$ or $B$ by $\infty$, the distribution is singly truncated from above or below respectively. 
The mean and variance of this doubly truncated normal variable $X$ are given by

$$
E(X)=\mu_{t}=\mu+\frac{\sigma\left\{\phi_{1}-\phi_{2}\right\}}{\Phi\left(\frac{B-\mu}{\sigma}\right)-\Phi\left(\frac{A-\mu}{\sigma}\right)}
$$

and

$$
V(X)=\sigma_{p}^{2}=\sigma^{2}\left[1+\frac{\left(\frac{A-\mu}{\sigma}\right) \phi_{1}-\left(\frac{B-\mu}{\sigma}\right) \phi_{2}}{\Phi\left(\frac{B-\mu}{\sigma}\right)-\Phi\left(\frac{A-\mu}{\sigma}\right)}-\left\{\frac{\phi_{1}-\phi_{2}}{\Phi\left(\frac{B-\mu}{\sigma}\right)-\Phi\left(\frac{A-\mu}{\sigma}\right)}\right\}^{2}\right]
$$

where $\phi_{1}=\phi\left(\frac{A-\mu}{\sigma}\right)$ and $\phi_{2}=\phi\left(\frac{B-\mu}{\sigma}\right)$.

We refer an interested reader to Schneider (1986), Johnson, Kotz and Balakrishnan (1994), Patel and Read (1996), Khasawneh et al. (2005 a, b), Cha, Cho and Sharp (2013), Ahsanullah et al. (2014), Cha and Cho (2014), for overview of the truncated normal distributions, their properties and applications and exhaustive updated bibliography.

\section{Assumptions and Notations}

In the development of the power of the control chart and $A R L$ for equation (1), the following assumptions are made and notations are used:

i. The measurement of items is considered to ascertain the magnitude of the variable characteristics in the lot.

ii. The process has doubly truncated normal distribution with mean $\mu_{t}$ and variance $\sigma_{p}^{2}$

iii. The applied measurement process (which is independent of the manufacturing process) has a variance $\sigma_{m}^{2}$. Thus, the overall variability is given by $\sigma^{2}=\sigma_{p}^{2}+\sigma_{m}^{2}$;

iv. Measurements of the items are taken to classify the produced units into defective and non-defective ones;

v. The process is in a state of statistical control at the time of determining the control limits and the same measuring instrument is used for later measurements;

vi. When the process parameter shifts, the data still comes restricted from DTND, however, with mean $\mu_{t^{\prime}}$ and variance $\left(\sigma_{p^{\prime}}^{2}+\sigma_{m}^{2}\right)$ where $\sigma_{p^{\prime}}^{2}$ is the process variance when the process parameter shifts (For details see Chakraborty and Khurshid, 2013 $\mathrm{a}, \mathrm{b})$.

Thus, considering the above assumptions, exact Shewhart control limits will be $\mu_{t} \pm K \sqrt{\sigma_{p}^{2}+\sigma_{m}^{2}}$. Normally we choose $K=3$ as it will give no false alarm with probability of at least $99.73 \%$ (Montgomery, 2013) and $n$ is the size of sample. The power of detecting the change of process parameter is given by

$$
P_{d}=P\left\{\bar{X} \geq \mu_{t}+3 \sqrt{\left(\sigma_{p}^{2}+\sigma_{m}^{2}\right) / n}\right\}+P\left\{\bar{X} \leq \mu_{t}-3 \sqrt{\left(\sigma_{p}^{2}+\sigma_{m}^{2}\right) / n}\right\}
$$




\section{Power of control chart for standardized doubly truncated normal distribution}

Instead of plotting measurement values in the control chart, we can standardize the variables as given below and plot accordingly (Khurshid and Chakraborty, 2014). This standardization procedure not only stabilizes the variables, but also stabilizes the resulting control chart. In this case the control limits as well as central lines are invariant with sample size $n$.

Thus, equation (4) can be expressed in terms of standardized normal variable $Z$ (when sample size is large and varies):

$$
Z \mid\left\{\left(\mu_{t^{\prime}}, \sigma_{p^{\prime}}^{2}, \sigma_{m}^{2}, n\right)\right\}=\frac{\bar{X}-\mu_{t^{\prime}}}{\sqrt{\left(\left(\sigma_{p^{\prime}}^{2}+\sigma_{m}^{2}\right) / n\right)}}
$$

Now, following Kanazuka (1986), Chakraborty and Khurshid (2013 b) and using equation (5), when the process parameter changes from $\mu_{t}$ to $\mu_{t^{\prime}}$, the power of the control chart for equation (1) is

$$
\begin{aligned}
& P_{\bar{X} \mid\left\{\left(\mu_{t}, \mu_{t^{\prime}}, \sigma_{p}^{2}, \sigma_{p^{\prime}}^{2}, \sigma_{m}^{2}, n\right)\right\}}=P_{d}=P\left\{\left(\bar{X} \mid \frac{\bar{X}-\mu_{t^{\prime}}}{\sqrt{\left(\sigma_{p^{\prime}}^{2}+\sigma_{m}^{2}\right) / n}} \geq \frac{\mu_{t}-\mu_{t^{\prime}}}{\sqrt{\left(\sigma_{p^{\prime}}^{2}+\sigma_{m}^{2}\right) / n}}+3 \frac{\sqrt{\sigma_{p}^{2}+\sigma_{m}^{2}}}{\sqrt{\left(\sigma_{p^{\prime}}^{2}+\sigma_{m}^{2}\right)}}\right)\right\} \\
& +P\left\{\left(\bar{X} \mid \frac{\bar{X}-\mu_{t^{\prime}}}{\sqrt{\left(\sigma_{p^{\prime}}^{2}+\sigma_{m}^{2}\right) / n}} \leq \frac{\mu_{t}-\mu_{t^{\prime}}}{\sqrt{\left(\sigma_{p^{\prime}}^{2}+\sigma_{m}^{2}\right) / n}}-3 \frac{\sqrt{\sigma_{p}^{2}+\sigma_{m}^{2}}}{\sqrt{\left(\sigma_{p^{\prime}}^{2}+\sigma_{m}^{2}\right)}}\right)\right\} \\
& =P\left\{\left(Z \mid Z \geq \frac{\mu_{t}-\mu_{t^{\prime}}}{\sqrt{\left(\sigma_{p^{\prime}}^{2}+\sigma_{m}^{2}\right) / n}}+3 \frac{\sqrt{\sigma_{p}^{2}+\sigma_{m}^{2}}}{\sqrt{\left(\sigma_{p^{\prime}}^{2}+\sigma_{m}^{2}\right)}}\right)\right\} \\
& +P\left\{\left(Z \mid Z \leq \frac{\mu_{t}-\mu_{t^{\prime}}}{\sqrt{\left(\sigma_{p^{\prime}}^{2}+\sigma_{m}^{2}\right) / n}}-3 \frac{\sqrt{\sigma_{p}^{2}+\sigma_{m}^{2}}}{\sqrt{\left(\sigma_{p^{\prime}}^{2}+\sigma_{m}^{2}\right)}}\right)\right\} \\
& \left.=P\left\{Z Z Z \geq \frac{\left(\frac{\mu_{t}-\mu_{t^{\prime}}}{\sigma_{p}}\right) \sqrt{n}}{\sqrt{\left(\sigma_{p^{\prime}}^{2} / \sigma_{p}^{2}\right)+\left(\sigma_{m}^{2} / \sigma_{p}^{2}\right)}}+3 \sqrt{\frac{1+\left(\sigma_{m}^{2} / \sigma_{p}^{2}\right)}{\left(\sigma_{p^{\prime}}^{2} / \sigma_{p}^{2}\right)+\left(\sigma_{m}^{2} / \sigma_{p}^{2}\right)}}\right)\right\} \\
& \left.+P\left\{Z Z \leq \frac{\left(\frac{\mu_{t}-\mu_{t^{\prime}}}{\sigma_{p}}\right) \sqrt{n}}{\sqrt{\left(\sigma_{p^{\prime}}^{2} / \sigma_{p}^{2}\right)+\left(\sigma_{m}^{2} / \sigma_{p}^{2}\right)}}-3 \sqrt{\frac{1+\left(\sigma_{m}^{2} / \sigma_{p}^{2}\right)}{\left(\sigma_{p^{\prime}}^{2} / \sigma_{p}^{2}\right)+\left(\sigma_{m}^{2} / \sigma_{p}^{2}\right)}}\right)\right\}
\end{aligned}
$$




$$
\begin{gathered}
\left.=P\left\{|Z| Z \geq \frac{-d \sqrt{n}}{\sqrt{\left(K^{2}+R^{2}\right)}}+3 \frac{\sqrt{1+R^{2}}}{\sqrt{\left(K^{2}+R^{2}\right)}}\right)\right\} \\
+P\left\{\left(Z \mid Z \leq \frac{-d \sqrt{n}}{\sqrt{\left(K^{2}+R^{2}\right)}}-3 \frac{\sqrt{1+R^{2}}}{\sqrt{\left(K^{2}+R^{2}\right)}}\right)\right\} \\
\left.=P\left\{|Z| Z \geq \sqrt{\frac{1+R^{2}}{\left(K^{2}+R^{2}\right)}}\left[3-\frac{d \sqrt{n}}{\sqrt{\left(1+R^{2}\right)}}\right]\right)\right\} \\
+P\left\{\left(Z \mid Z \leq \sqrt{\frac{1+R^{2}}{\left(K^{2}+R^{2}\right)}}\left[-3-\frac{d \sqrt{n}}{\sqrt{\left(1+R^{2}\right)}}\right]\right)\right\} \\
=\Phi\left\{\sqrt{\frac{1+R^{2}}{\left(K^{2}+R^{2}\right)}}\left(-3+\frac{d \sqrt{n}}{\sqrt{1+R^{2}}}\right)\right\}+\Phi\left\{\sqrt{\frac{1+R^{2}}{\left(K^{2}+R^{2}\right)}}\left(-3-\frac{d \sqrt{n}}{\sqrt{1+R^{2}}}\right)\right\} \\
=\Phi(M)+\Phi(N)
\end{gathered}
$$

where $\left.d=\left\{\left(\mu_{t^{\prime}}-\mu_{t}\right) / \sigma_{p}\right)\right\}, K^{2}=\sigma_{p^{\prime}}^{2} / \sigma_{p}^{2}, R^{2}=\sigma_{m}^{2} / \sigma_{p}^{2}$,

$$
\begin{aligned}
& M=\left\{\sqrt{\frac{1+R^{2}}{\left(K^{2}+R^{2}\right)}}\left(-3+\frac{d \sqrt{n}}{\sqrt{1+R^{2}}}\right)\right\}, N=\left\{\sqrt{\frac{1+R^{2}}{\left(K^{2}+R^{2}\right)}}\left(-3-\frac{d \sqrt{n}}{\sqrt{1+R^{2}}}\right)\right\} \text { and } \\
& \Phi(z)=\frac{1}{\sqrt{2 \pi}} \int_{-\infty}^{z} e^{-\left(u^{2} / 2\right)} d u .
\end{aligned}
$$

Using Equation (6), the power of the control chart $P_{d}$ can be obtained easily by solving $\Phi(z)$ for different combinations of $d, K^{2}$ and $R^{2}$, as shown in Tables 1-6.

\section{Average Run Length ( $A R L$ ) for DTND under measurement error}

To study the sensitivity of the monitoring procedure, one can also study $A R L$ which is the average number of points that must be plotted before a point indicates an out of control condition when operating is statistical control (Khurshid and Chakraborty, 2014).

For any Shewhart control chart, the $A R L=[P]^{-1}$ where $P$ is the probability that a single point exceeds the control limits. Thus instead of drawing conclusion based on $P_{\bar{X}}$, one can interpret the results of the power of control chart in terms of $A R L$ just by reversing equation (6) i.e., in this case

$$
A R L=\left[\Phi\left\{\sqrt{\frac{1+R^{2}}{\left(K^{2}+R^{2}\right)}}\left(-3+\frac{d \sqrt{n}}{\sqrt{1+R^{2}}}\right)\right\}+\Phi\left\{\sqrt{\frac{1+R^{2}}{\left(K^{2}+R^{2}\right)}}\left(-3-\frac{d \sqrt{n}}{\sqrt{1+R^{2}}}\right)\right\}\right]^{-1}
$$


The values of $A R L$ are shown in Table 7.

\section{Concluding Remarks}

The effects of truncation as well as measurement errors on the power of detecting the changes in the process parameters by $3 \sigma$ control limits with the control chart for DTND are shown in Tables 1-7.

It has been observed from Table 1 that as we go on increasing the shift of the process parameter $\mu_{t}$ to $\mu_{t^{\prime}}$ there is an increase in the power of control chart $P_{d}$ for fixed values of $n, \mu, \sigma, \sigma_{m}$ and truncation points $A$ and $B$. It can also be concluded that as the ratio between $\mu_{t}$ to $\mu_{t^{\prime}}$ decrease there is an increasing trend in the values of $P_{d}$ the power of control chart.

Unlike other discrete distributions, as studied by Khurshid and Chakraborty (2014), the smaller values of $K^{2}$ corresponds to the smaller values of $P_{d}$.

From the Tables 1 and 2, it has been observed that the values of $P_{d}$ considerably increase as we go on increasing the values of $n$ for fixed $\mu, \sigma, \sigma_{m}$ and truncation points $A$ and $B$.

Tables 2 and 3 show that the values of $P_{d}$ decrease as we go on increasing the values of $\sigma_{m}$ for fixed $n, \mu, \sigma$ and truncation points $A$ and $B$.

For fixed $d$, Table 4 shows the values of $P_{d}$ which increases as the ratio to $\mu_{t}$ to $\mu_{t^{\prime}}$ decreases.

From the Tables 1 and 5, it has been observed that as we increase the range of truncations, there is a decrease in the values of $P_{d}$ when the ratios of $\mu_{t}$ to $\mu_{t^{\prime}}$ are same.

Table 6 shows the values of $P_{d}$ corresponds to the value of $\mu$. As we go on increasing $\mu$ there is an increasing trend in the values of $P_{d}$. However the values of $P_{d}$ are more relevant if the ratio of $\mu_{t}$ to $\mu_{t^{\prime}}$ is much smaller.

It has also been observed from the above tables that relative measurement errors $\left(R^{2}\right)$ tend to increase as we increase the values of $\sigma_{m}$. The corresponding values of $P_{d}$ decreases when the values of $R^{2}$ increase.

Table 7 gives the values of $A R L$. It has been observed from the table that $A R L$ values increase as there is an increase in the range of truncation. Also $A R L$ values increase as there is an increase in the values of $R^{2}$ for fixed $\mu$ and $\sigma$. 


\section{References}

1. Ahsanullah, M., Kibria, B. M. G. and Shakil, M. (2014). Normal and Student's t distributions and their Applications. Paris, France: Atlantis Press.

2. Cha, J. and Cho, B. R. (2014). Classical statistical inference extended to truncated populations for continuous process improvement: Test statistics, p-values, and confidence intervals. Quality and Reliability Engineering International, DOI:10.1002/qre.1719.

3. Cha, J., Cho, B. R. and Sharp, J. L. (2013). Rethinking the truncated normal distribution. International Journal of Experimental Design and Process Optimisation, 3, 327-363.

4. Chakraborty, A. B. and Khurshid, A. (2013 a). Measurement error effect on the power of control chart for zero-truncated Poisson distribution. International Journal for Quality Research, 7, 411-419.

5. Chakraborty, A. B. and Khurshid, A. (2013 b). Measurement error effect on the power of control chart for the ratio of two Poisson distributions. Economic Quality Control, 28, 15-21.

6. Cohen, A. (1949). On estimating the mean and standard deviation of truncated normal distributions. Journal of the American Statistical Association, 44, 518525.

7. Cohen, A. (1950). Estimating the mean and variance of normal populations from singly truncated and doubly truncated samples. Annals of Mathematical Statistics, 21, 557-569.

8. Cohen, A. (1991). Truncated and Censored Samples: Theory and Applications. New York: Marcel Dekker.

9. Cox, M. A. A. (2009). Control charts for monitoring observations from a truncated normal distribution. The Journal of Risk Finance, 10, 288-304.

10. Horrace, W. C. (2915). Moments of the truncated normal distribution. Journal of Productivity Analysis, 43, 133-138.

11. Johnson, A. (2001). On the truncated normal distribution: Characteristics of singly- and doubly-truncated populations of application in management science. Doctoral Dissertation, Stuart Graduate School of Business, Illinois Institute of Technology, Chicago, Illinois.

12. Johnson, A. C. and Thomopoulos, N. T. (2004). Characteristics and tables of the left-truncated normal distribution. Proceedings of the Midwest Decision Sciences Institute, Cleveland, pp. 133-139.

13. Johnson, N. L., Kotz, S., and Balakrishnan, N. (1994). Continuous Univariate Distributions, Volume 1, Second Edition. New York: John Wiley and Sons.

14. Kakoty, S, and Chakraborty, A. B. (1990). A continuous acceptance sampling plan for truncated normal distribution based on cumulative scores. Quality Reliability, 2, 15-18. 
15. Kanazuka, T. (1986). The effects of measurement error on the power of $\bar{X}-R$ charts. Journal of Quality Technology, 18, 91-95.

16. Kendall, M. G. and Stuart, A. (1979). The Advanced theory of Statistics, Vol 2, Fourth Edition. London: Charles Griffin and Company.

17. Khasawneh, M. T., Bowling, S. R., Kaewkuckool, S. and Cho, B. R. (2005 a). Tables of a truncated normal distribution: A singly truncated case. Quality Engineering, 17, 33-50.

18. Khasawneh, M. T., Bowling, S. R., Kaewkuckool, S. and Cho, B. R. (2005 b). Tables of a truncated normal distribution: A doubly truncated case. Quality Engineering, 17, 227-241.

19. Khurshid, A. and Chakraborty, A. B. (2014). Measurement error effect on the power of control chart for zero-truncated binomial distribution under standardization procedure. International Journal for Quality Research, 8, 495504.

20. Montgomery, D. C. (2013). Introduction to Statistical Quality Control, Seventh Edition. New York: John Wiley and Sons.

21. Patel, J. K. and Read, C. B. (1996). Handbook of the Normal Distribution, Second Edition (Revised and Expanded). Marcel Dekker: New York.

22. Rai, G. (1966). Cumulative sum control charts for truncated normal distribution. Australian and New Zealand Journal of Statistics, 8, 80-86.

23. Ryan, T. P. (2011). Statistical Methods for Quality Improvement, Third Edition. New York: John Wiley and Sons.

24. Sankle, R., Singh, J. R. and Mangal, I. K. (2012). Cumulative sum control charts for truncated normal distribution under measurement error. Statistics in Transition (New Series), 13, 95-106.

25. Schneider, H. (1985). The performance of variable sampling plans when the normal distribution is truncated. Journal of Quality Technology, 17, 74-80.

26. Schneider, H. (1986). Truncated and Censored Samples from Normal Populations. Marcel Dekker: New York. 
Table 1: Values of $P_{d}$ for controlling the parameter $\lambda$

When $A=15, \quad B=40, \quad n=5, \quad \mu=0.5, \sigma=10, \sigma_{m}=2, \quad \mu_{t}=24.46, \quad \sigma_{p}=6.135$, $R^{2}=0.11$

\begin{tabular}{|c|c|c|c|c|c|c|}
\hline$\mu_{t^{\prime}}$ & $\sigma_{p^{\prime}}$ & $\left.d=\left\{\left(\mu_{t^{\prime}}-\mu_{t}\right) / \sigma_{p}\right)\right\}$ & $K^{2}$ & $\Phi(M)$ & $\Phi(N)$ & $P_{d}$ \\
\hline 25.0 & 6.20 & 0.09 & 1.02 & 0.0027 & 0.0008 & 0.0035 \\
\hline 25.5 & 6.30 & 0.17 & 1.05 & 0.0049 & 0.0005 & 0.0054 \\
\hline 26.0 & 6.40 & 0.25 & 1.08 & 0.0084 & 0.0003 & 0.0087 \\
\hline 26.5 & 6.50 & 0.33 & 1.12 & 0.0146 & 0.0001 & 0.0147 \\
\hline 27.0 & 7.00 & 0.41 & 1.30 & 0.0294 & 0.0004 & 0.0253 \\
\hline
\end{tabular}

Table 2: Values of $P_{d}$ for controlling the parameter $\lambda$

\begin{tabular}{|c|c|c|c|c|c|c|}
\hline \multicolumn{7}{|c|}{$\begin{array}{l}\text { When } A=15, \quad B=40, \quad n=10, \quad \mu=20, \sigma=10, \sigma_{m}=2, \quad \mu_{t}=24.46, \sigma_{p}=6.135, \\
R^{2}=0.11\end{array}$} \\
\hline$\mu_{t^{\prime}}$ & $\sigma_{p^{\prime}}$ & $\left.d=\left\{\left(\mu_{t^{\prime}}-\mu_{t}\right) / \sigma_{p}\right)\right\}$ & $K^{2}$ & $\Phi(M)$ & $\Phi(N)$ & $P_{d}$ \\
\hline 25.0 & 6.20 & 0.09 & 1.02 & 0.0034 & 0.0006 & 0.0040 \\
\hline 25.5 & 6.30 & 0.17 & 1.05 & 0.0075 & 0.0004 & 0.0079 \\
\hline 26.0 & 6.40 & 0.25 & 1.08 & 0.015 & 0 & 0.015 \\
\hline 26.5 & 6.50 & 0.33 & 1.12 & 0.028 & 0 & 0.028 \\
\hline 27.0 & 7.00 & 0.41 & 1.30 & 0.059 & 0 & 0.059 \\
\hline
\end{tabular}

Table 3: Values of $P_{d}$ for controlling the parameter $\lambda$

When $A=15, B=40, n=10, \quad \mu=20, \sigma=10, \sigma_{m}=4, \quad \mu_{t}=24.46, \sigma_{p}=6.135$, $R^{2}=0.43$

\begin{tabular}{|c|c|c|c|c|c|c|}
\hline$\mu_{t^{\prime}}$ & $\sigma_{p^{\prime}}$ & $\left.d=\left\{\left(\mu_{t^{\prime}}-\mu_{t}\right) / \sigma_{p}\right)\right\}$ & $K^{2}$ & $\Phi(M)$ & $\Phi(N)$ & $P_{d}$ \\
\hline 25.0 & 6.20 & 0.09 & 1.02 & 0.0019 & 0.0011 & 0.0030 \\
\hline 25.5 & 6.30 & 0.17 & 1.05 & 0.0060 & 0.0003 & 0.0063 \\
\hline 26.0 & 6.40 & 0.25 & 1.08 & 0.0087 & 0 & 0.0087 \\
\hline 26.5 & 6.50 & 0.33 & 1.12 & 0.0207 & 0 & 0.0207 \\
\hline 27.0 & 7.00 & 0.41 & 1.30 & 0.0409 & 0 & 0.0409 \\
\hline
\end{tabular}

Table 4: Values of $P_{d}$ for controlling the parameter $\lambda$

\begin{tabular}{|c|c|c|c|c|c|}
\hline \multicolumn{6}{|c|}{$\begin{array}{l}\text { When } A=15, B=40, n=5, \mu=20, \sigma=10, \sigma_{m}=2, \mu_{t}=24.46 \text {, } \\
\left.R^{2}=0.11, d=\left\{\left(\mu_{t^{\prime}}-\mu_{t}\right) / \sigma_{p}\right)\right\}=0.09\end{array}$} \\
\hline$\mu_{t^{\prime}}$ & $\sigma_{p^{\prime}}$ & $K^{2}$ & $\Phi(M)$ & $\Phi(N)$ & $P_{d}$ \\
\hline 25.0 & 6.20 & 1.02 & 0.0027 & 0.0008 & 0.0035 \\
\hline 25.5 & 6.30 & 1.05 & 0.0030 & 0.0009 & 0.0039 \\
\hline 26.0 & 6.40 & 1.08 & 0.0034 & 0.001 & 0.0044 \\
\hline 26.5 & 6.50 & 1.12 & 0.0038 & 0.0021 & 0.0059 \\
\hline 27.0 & 7.00 & 1.30 & 0.0064 & 0.0023 & 0.0087 \\
\hline
\end{tabular}


Table 5: Values of $P_{d}$ for controlling the parameter $\lambda$

When $A=15, B=40, \quad n=5, \quad \mu=20, \sigma=10, \quad \sigma_{m}=2, \quad \mu_{t}=22.30, \quad \sigma_{p}=8.47$, $R^{2}=0.06$

\begin{tabular}{|c|c|c|c|c|c|c|}
\hline$\mu_{t^{\prime}}$ & $\sigma_{p^{\prime}}$ & $\left.d=\left\{\left(\mu_{t^{\prime}}-\mu_{t}\right) / \sigma_{p}\right)\right\}$ & $K^{2}$ & $\Phi(M)$ & $\Phi(N)$ & $P_{d}$ \\
\hline 22.79 & 8.560 & 0.06 & 1.02 & 0.0023 & 0.0001 & 0.0024 \\
\hline 23.25 & 8.698 & 0.11 & 1.05 & 0.0027 & 0.0006 & 0.0033 \\
\hline 23.70 & 8.836 & 0.17 & 1.08 & 0.0055 & 0.0006 & 0.0061 \\
\hline 24.16 & 8.994 & 0.22 & 1.12 & 0.0087 & 0.0005 & 0.0092 \\
\hline 24.62 & 9.664 & 0.27 & 1.30 & 0.0166 & 0.0008 & 0.0174 \\
\hline
\end{tabular}

Table 6: Values of $P_{d}$ for controlling the parameter $\lambda$

\begin{tabular}{|c|c|c|c|c|c|c|}
\hline $\begin{array}{l}\text { When } \\
R^{2}=0.05\end{array}$ & $5, \quad B$ & $0, \quad n=5, \quad \mu=$ & $=10$, & 2 , & 45, & $=6.45$, \\
\hline$\mu_{t^{\prime}}$ & $\sigma_{p^{\prime}}$ & $\left.d=\left\{\left(\mu_{t^{\prime}}-\mu_{t}\right) / \sigma_{p}\right)\right\}$ & $K^{2}$ & $\Phi(M)$ & $\Phi(N)$ & $P_{d}$ \\
\hline 27.0339 & 6.5183 & 0.090 & 1.02 & 0.0027 & 0.0008 & 0.0035 \\
\hline 27.5746 & 6.6235 & 0.170 & 1.05 & 0.0049 & 0.0005 & 0.0054 \\
\hline 28.1153 & 6.7286 & 0.258 & 1.08 & 0.0091 & 0.0003 & 0.0094 \\
\hline 28.6560 & 6.8337 & 0.342 & 1.12 & 0.0154 & 0.0001 & 0.0155 \\
\hline 29.1966 & 7.3594 & 0.426 & 1.30 & 0.0322 & 0.0003 & 0.0325 \\
\hline
\end{tabular}

Table 7: Values of $A R L$

\begin{tabular}{|c|c|c|c|c|}
\hline \multicolumn{5}{|c|}{$A=15, B=40, \mu=20, \sigma=10$} \\
\hline$d$ & $K^{2}$ & $R^{2}$ & $n$ & $A R L$ \\
\hline 0.09 & 1.02 & 0.11 & 10 & 250 \\
\hline & & 0.11 & 5 & 285.71 \\
\hline & & 0.43 & 10 & 333.33 \\
\hline \multicolumn{5}{|c|}{$A=10, B=40, \mu=25, \sigma=10$} \\
\hline 0.06 & 1.02 & 0.06 & 5 & 416.67 \\
\hline
\end{tabular}

